\title{
Ovarian fibroma in Young Women with Infertility Mimicking Vterine fibroid
}

\section{JGR}

\section{Rajshree Dayanand Katke}

Department of Obstetrics \& Gynecology, Cama \& Albless Hospital, Mumbai-400001,

Maharashtra, India.

\section{Abstract:}

Ovarian fibroma represents $4 \%$ of all ovarian neoplasm's occurring mostly in peri and post menopausal women $[1,2]$. They are often difficult to diagnose preoperatively and commonly misdiagnosed as uterine fibromas, because of their same pathology, complications, clinical and ultrasonic features [3,4], or sometimes as malignant ovarian tumors because of accompanying ascites and increased serum CA-125 level [5]. This is rare case of ovarian fibroma misdiagnosed as uterine fibroid in young women. This case highlights the diagnostic difficulties that may be encountered in the management of ovarian fibroma including potential misdiagnosis of the tumor as uterine fibroid that may influence the surgical approach.

Key words: Ascites, CA-1 25 Antigen, Fibroma, Leiomyoma, Ovarian Neoplasms.

\section{Introduction}

Ovarian fibromas are benign ovarian tumors of sex cord stromal origin. Although fibromas account for approximately $4 \%$ of all ovarian neoplasms, they are the most common sex cord ovarian tumor. Ovarian fibroma is generally asymptomatic and seen in women $>50$ years of age. However, abdominal pain and acute abdomen secondary to torsion may be presenting feature [6]. Diagnosis is usually made by ultrasonography showing a solid ovarian lesion, or, on some occasions, mixed tumors with solid and cystic components [2]. Computed tomography and magnetic resonance imaging can also be used to diagnose fibromas. We hereby present a case of a 30 year old young woman who presented with abdominal pain and secondary infertility. Ultrasound evaluation misdiagnosed it as uterine fibroid. The patient underwent exploratory laparotomy with ovarian mass excision accompanied by frozen section which revealed it as ovarian fibroma.

\section{Case Report}

32 year old para 1 female presented with abdominal pain since six months and secondary infertility since 6 years. Patient had history of irregular menses and heavy menses since last 6 months. Her previous menses were regular with moderate bleeding. There was no history of anorexia, evening rise of temperature and weight loss. There was no significant past history of any medical or surgical illness. On examination patient's

\section{Corresponding Author: Dr. Rajshree Dayanand Katke} Email: drrajshrikatke@gmail.com

Received: December 7, 2015 | Accepted: February 20, 2016 | Published Online: March 20, 2016 This is an Open Access article distributed under the terms of the Creative Commons Attribution License (creativecommons.org/licenses/by/3.0) Conflict of interest: None declared | Source of funding: Nil | DOI: http://dx.doi.org/10.17659/01.2016.0029 
general condition was fair with average built, her vitals were within normal limits and no abnormality was detected in respiratory, cardiovascular system or central nervous system. A 12 weeks pregnancy size mass was found on abdominal examination with no guarding, tenderness or rigidity.

Per speculum examination showed healthy cervix and vagina. Per vaginal examination was suggestive of uterus corresponding to 18-20 weeks of gestation size with free and nontender bilateral fornices. Ultrasound examination showed large lesion in pelvis of size $15 \times 10.4 \mathrm{~cm}$ not separate from fundus suggestive of subserosal fundal fibroid with few small multiple subserosal fibroid on anterior and posterior wall of uterus. Bilateral ovaries were normal. Hysterosalpingography showed bilateral patent tubes. Her all routine investigations were within normal limits. Patient was posted for exploratory myomectomy with chromopertubation. On the contrary to ultrasound examination in situ, there was greyish white coloured oval mass of size $15 \times 11 \mathrm{~cm}$ size arising from left ovary positioned over the normal sized uterus with two small $2 \times 2 \mathrm{~cm}$ subserosal fibroid [Fig.1,2]. Left ovary was not seen separately from the mass, and right ovary was normal. Rest of the abdominal organs were normal. Ovarian mass was excised and sent for frozen section. Gross specimen revealed oval shaped well encapsulated, greyish white, firm ovarian mass $15 \times 12 \times 8 \mathrm{~cm}$ [Fig.3]. The cut surface was greywhite and homogeneous with a whorled pattern and occasional areas of calcification [Fig.4]. Report of frozen section was suggestive of ovarian fibroma. Immediate postoperative CA 125 level was $5 \mathrm{IU} / \mathrm{ML}$. Bilateral chromopertubation showed normal spill on both sides. Microscopic features revealed tumor cells arranged in diffuse sheets and at places showing sporiform pattern. Individual tumor cells were spindle shaped with moderate amount of eosinophillic cytoplasm with oval to spindle nuclei. All these findings in final HPR confirmed diagnosis of ovarian fibroma. Post-operatively patient recovered well and was discharged in healthy condition.
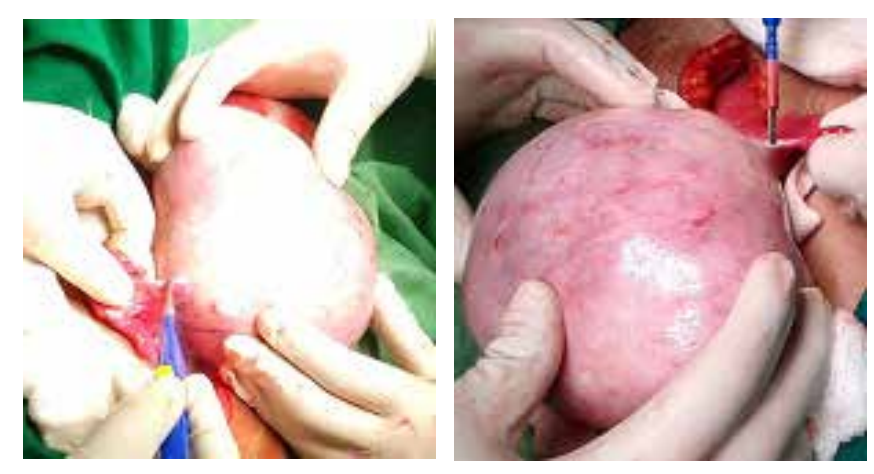

Fig.1,2: In situ appearance of ovarian fibroma.

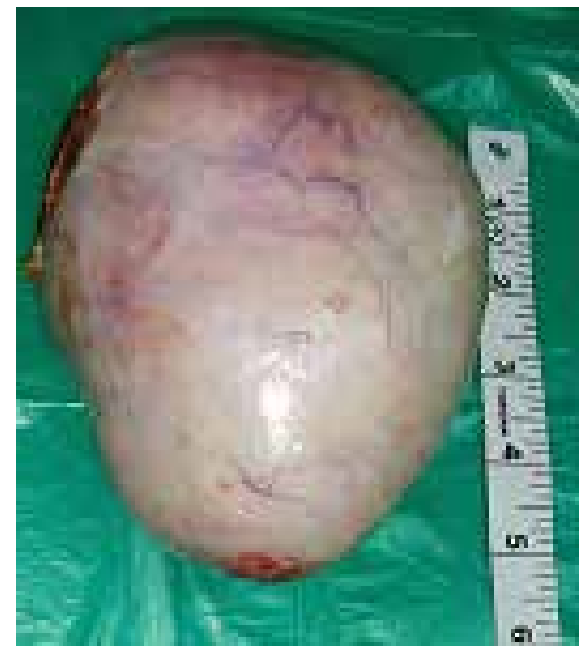

Fig.3: Gross appearance of ovarian fibroma.

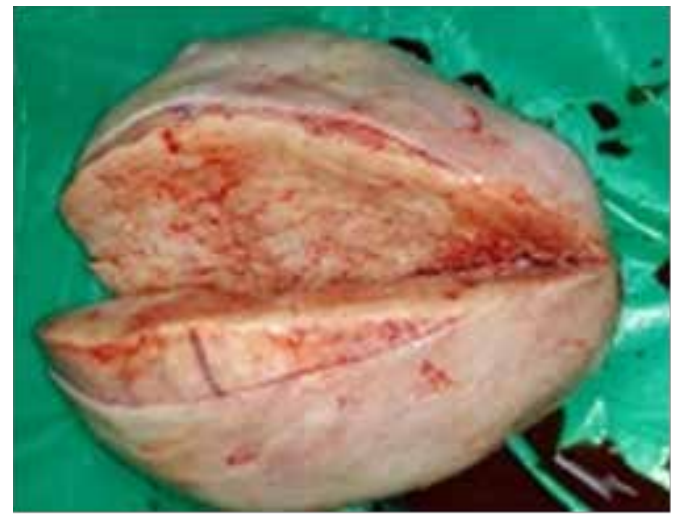

Fig.4: Cut section of ovarian fibroma. 


\section{Discussion}

Mesenchymal neoplasms of the ovary are uncommon, not specific and determine difficult problems in diagnosis, histogenesis, behavior, and therapy [7]. Ovarian fibroma can be bilateral in $4 \%-8 \%$ of patients and multiple in $10 \%$ of cases [7], especially in Gorlin syndrome [9], or associated with pleural effusion and ascites in Meigs' syndrome [9]. It is often seen concomitantly with uterine leiomyoma suggesting an identical hormonal stimulation and rarely associated with hirsutism or infertility. Sometimes it may present as huge ovarian mass with torsion requiring emergency surgical intervention [6].

Ovarian fibromas are stromal tumors composed of spindle, oval or round cells producing collagen. Fibromas are usually solid, spherical, slightly lobulated, encapsulated, grey-white masses covered by a glistening, intact ovarian serosa. Ovarian fibromas are almost always benign. Very rarely, fibromas without any atypical features are associated with peritoneal implants. Ultrasound examination, CT scan, MRI and tumor markers like CA-125, CEA, AFP help in diagnosis. Because of its rarity, the immunohistochemical analysis with desmin, inhibin -smooth muscle actin or histochemical staining with Masson's trichrome is recommended to rule out the differential diagnosis especially leiomyosarcoma and sex-cord stromal tumors (thecoma and sclerosing stromal tumor).

Surgery is the recommended treatment for ovarian fibroma. Salpingo-oophorectomy can be considered in perimenopausal or postmenopausal women, and cystectomy only can be performed in young women. Exploratory laparotomy is standardized approach but laparoscopic surgery is safe alternative approach provided preoperatively diagnosis is confirmed.

\section{Conclusion}

Our case emphasises that ovarian fibromas though very rare in young patients should be preoperatively considered in the differential diagnosis and investigated thoroughly. Its treatment requires surgical excision with frozen section. Regular follow up should be kept in view of recurrence.

\section{References}

1. Sheila Jorge Adad, Valeria Lima Laterza, Carlos David Teixeira dos Santos, et al. Cellular Fibroma of the Ovary with Multiloculated Macroscopic Characteristics: Case Report. Case Reports in Medicine, vol. 2012, Article ID 283948, 5 pages, 2012.

2. Yen P. Khong K, Lamba, R, Corwin, MT, Gerscovich, EO. Ovarian fibromas and fibrothecomas: Sonographic correlation with computed tomography and magnetic resonance imaging: J Ultrasound Med. 2013;32(1):13-18.

3. Gogineni S, Sunitha $C$, Chelamkuri S. Extrauterine Postmenopausal fibroid with torsion. Journal of Dental and Medical Sciences. 2013;5(5):6971.

4. Najmi Z, Mehdizadehkashi A, Kadivar $M$, Tamannaie Z, Chaichian S. Laparoscopic Approach to a Large Ovarian Fibroma: A Case Report. J Reprod Infertil. 2014;15(1):57-60.

5. Macciò A, Madeddu C, Kotsonis P, Pietrangeli $M$, Paoletti AM. Large twisted ovarian fibroma associated with Meigs' syndrome, abdominal pain and severe anemia treated by laparoscopic surgery. BMC Surgery. 2014;14:38.

6. Katke RD. Torsion of a Huge Ovarian Fibroma Presented as Acute Abdomen. Journal of Case Reports. 2015;5:195-198.

7. Cambruzzi E, Pegas KL, Milani DM, Cruz RP, Guerra EH, Ferrari MB. Angiosarcoma arising in an ovarian fibroma: a case report. Patholog Res Int. $2010 ; 2010: 842592$. 
8. Aram S, Moghaddam NA. Bilateral ovarian fibroma associated with Gorlin syndrome. JRMS. 2008; 1 4(1):57-61.

9. Yazdani S, Alijanpoor A, Sharbatdaran $M$, Bouzari Z, Abedisamakoosh M, Lakaieandi
F, Mohammadpour M. Meigs' syndrome with elevated serum CA125 in a case of ovarian fibroma/thecoma. Caspian Journal of Internal Medicine. 2014;5(1):43-44. 